\title{
Commercial access for UK/ESA student experiments on board the ISS
}

\author{
Christopher J. Gaffney \\ Lancaster University \\ Lancaster, UK
}

\author{
Roberta Torregrossa, Colleen S. Deane, Matt \\ Whiteman, Timothy Etheridge \\ University of Exeter \\ Exeter, UK
}

\author{
Ramon Nartallo, Gianluca Neri, David Zolesi \\ Kayser Space \\ Didcot, UK
}

\author{
Rebecca A. Ellwood, Michael Cooke, Nima \\ Gharahdaghi, Mathew Piasecki, Bethan Phillips, \\ Nathaniel J. Szewczyk \\ University of Nottingham \\ Derby, UK \\ Nathaniel.Szewczyk@nottingham.ac.uk
}

\begin{abstract}
School students in the US have the ability to commercially fly experiments on-board the International Space Station (ISS) via programmes like the Nanoracks sponsored Student Spaceflight Experiment Program (SSEP). Programs like SSEP do allow international schools to participate but similar programmes do not currently exist within the European Space Agency (ESA). ESA does, however, support commercial access to space via companies like Airbus and Kayser Italia. A key principle of SSEP is that students propose to fly experiments that will work within existing spaceflight hardware. This is similar to the idea of using standardized CubeSat platforms in education and ESA's long-standing use of standardized Experiment Containers (ECs). These ECs form the starting point for Airbus and Kayser Italia's commercial access programmes. In 2018 we were selected by the UK Space Agency to develop and fly a UK national payload to the ISS. This payload will conduct scientific experiments proposed by ourselves, international partners, and schools in the UK. All experiments will take place inside ECs that are refurbished, and flight qualified in the UK. If we can successfully conduct student experiments during this mission, we will have demonstrated the possibility of conducting UK student experiments in space via a UK company. This should pave the way for UK-based commercial access to the ISS that could be used by schools much like the US based SSEP.
\end{abstract}

Keywords-Student spaceflight; experimental cassette; commercial spaceflight; CubeSat; schools

\section{INTRODUCTION TO STUDENT SPACEFLIGHT EXPERIMENTS}

Since June 2010, students in the US have had commercial access to space and the International Space Station (ISS) through programmes such as the Nanoracks-sponsored Student Spaceflight Experiment Program (SSEP). Platforms such as Nanoracks can provide regular access to space on the condition that the financial costs can be met. The SSEP is a model US National Science, Technology, Engineering, and Mathematics (STEM) initiative for 5-16 year old students, which is intended to inspire the next generation of America's Scientists and Engineers (1). It is the product of a joint partnership between the National Center for Earth and Space Science Education (NCESSE) and Nanoracks, LLC. Each round typically engages three hundred or more students, and they compete in local Flight Experiment Design competitions. The competition runs in a manner highly similar to that of grant proposals for scientists and thus, is an accurate depiction of future research as a space life scientist in the planning, feasibility and safety testing, and actual flight of an experiment.

\section{US-BASED STUDENT SPACEFLIGHT EXPERIMENT PROVISION: HOW IT WORKS}

In the US, individual school districts apply to host an SSEP, at a cost of $\$ 25,000$ (1). The basic model for these programmes is to pay money for access, design, and to fly a spaceflight experiment. Each district can secure a 'mini-lab' thereby obtaining the platform to fly the experiment in space, led by the experiment design competition. The SSEP provides all teaching and student resources and other resources for the engagement of the community including conference attendance or museum visits [1]. All experiments proposed, similar to research-led experiments, must pass a safety review to ensure that the experiment and samples pose no risk to the crew, resupply vehicles, or the ISS. Indeed, the level of risk permitted depends upon the toxicity of samples and how well they are contained, and this highlights the benefits of Nanoracks in the US and Experimental Cassettes (ECs) in Europe as a standardised platform, which provides protection of experiments for use in spaceflight research.

\section{A BRIEF HISTORY OF LIFE SCIENCES FLIGHT FACILITIES DEVELOPED FOR THE ISS}

Facilities originally designed for use on the ISS were to host experiments in evolutionary biology, developmental biology, gravitational ecology, cellular and molecular biology, and organismal and comparative biology [2]. Opportunities have expanded on the ISS to accommodate research in cell culture, an insect habitat and an animal habitat for rodents, an aquatic 
facility (small fish and aquatic specimens), a specialised egg incubator (developmental biology), and even a platform for research with small plants (European Modular Cultivation System (EMCS)) [3]. Habitats provide food and water, light, air, waste management, and provide control of both temperature and humidity for a multitude of organisms [3]. Broadly, these habitats have been established to investigate the effects of spaceflight on physiology. Spaceflight is associated with many physiological stressors including microgravity, radiation, changes in tensions of oxygen, carbon dioxide, and nitrogen. In order to determine the independent effects of microgravity on biological experiments in space, it has been necessary to develop centrifuges for application on the space station. The presence of a centrifuge and comparison to ground reference controls, allows elucidation of the effects of launch/landing accelerations, vibration, microgravity, and radiation [4]. Student-led experiments using an in-flight centrifuge such as Kubik are now a commercial reality in Europe through the use of ECs developed by Kayser Italia.

\section{THE DEVELOPMENT OF STUDENT LED EXPERIMENTS THAT FIT WITHIN THIS SETUP}

Physical space onboard the ISS is finite, therefore life science experiments and particularly those proposed by SSEP must be space efficient. Experiments designed by students using the Nanoracks platform, for example, can use one of three fluid mixing enclosures, specifically designed as hardware for spaceflight research [5]. There is typically a short turnaround between selection of proposals and flight (c. six months) limiting opportunities for validation of equipment and science verification testing [5]. European-led biological and life science experiments in space take place in incubators including Kubik, Biobox, EMCS, and BioLab [4].

These European incubators can house ECs (Fig 1) in a single temperature-controlled environment where some samples are at $0 \mathrm{~g}$ and others can be maintained at $1 \mathrm{~g}$ through an integrated centrifuge [6]. These incubators permit full automation of experiments during ISS flights, making it time efficient and little imposition for crew. ECs have been designed that slot into spaces within either Biobox or Kubik and they can be supplied with power to manipulate experimental conditions. Indeed, Kayser Italia has developed thirteen experimental units for use in space, which allow the culture of bacteria, cells, tissue culture, model organisms including C. elegans, and plants [6].

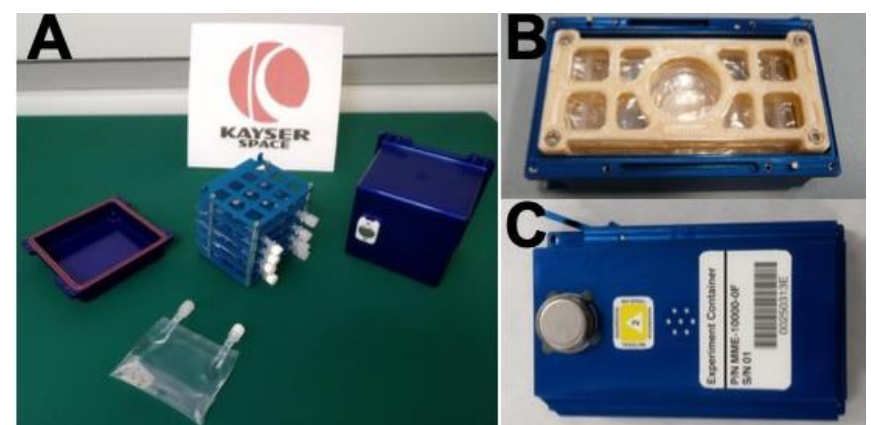

Fig 1. ECs suitable for use in student spaceflight experiments. (A) EC developed for the ESA Rotifer Investigation and $(B)$ open and $(C)$ closed image of the MME EC. Photo credit Kayser Space.

\section{BENEFITS OF STUDENT SPACEFLIGHT EXPERIMENTS FOR ENGAGEMENT IN STEM}

Student spaceflight experiment programmes are valuable for education outreach activities through inspiring the uptake of Science, Technology, Engineering, and Mathematics (STEM). Furthermore, they also help stimulate the next generation to take careers in STEM subjects and are the future of the space industry, including spaceflight research. Whilst programmes such as the SSEP do allow international students to participate, they are not widely advertised within European countries, and thus, uptake in these programmes from European countries is poor. Both the UK and Europe have our own respective Space Agencies, so it is no less important to inspire our future Scientists than in the USA.

\section{A EUROPEAN PLATFORM FOR STUDENT SPACEFLIGHT EXPERIMENTS}

ESA currently offers the Fly your thesis! programme, which offers European students the opportunity to design, build, and fly an experiment as part of a Masters or PhD thesis [7]. These experiments are then implemented on either a parabolic flight, a drop tower, a centrifuge, or a CubeSat [7]. ESA does support commercial spaceflight opportunities through companies like Airbus and Kayser Italia, however, the development of spaceflight hardware is a significant cost and prohibitively so for student projects. Thus, all experiments proposed in the USA programmes must work within existing flight hardware. In Europe, companies such as Kayser Italia (http://www.bioreactorexpress.space) and Airbus (http://www.kiwi-microgravity.com) have used standardised ECs for some time $[8,9]$. This EC platform has made access to space a commercial reality within Europe, similar to Nanoracks in the US. The EC is an aluminium box of approximately $20 \mathrm{x}$ $40 \times 80 \mathrm{~mm}$ (Fig 1). Just about any experiment that can fit within this box can be flown. As mentioned above, existing hardware allows the ECs to be flown with or without power, in or not in a temperature-controlled incubator and using a variety of previously developed hardware inserts. We have recently used this platform for the Molecular Muscle Experiment [10] and have recently been granted one of the first UK national payloads to fly to the ISS. In our current flight project, Molecular Muscle Experiment-2, we have been granted permission to fly three student led experiments within these ECs. If we can successfully conduct student experiments during this mission, we will have demonstrated the feasibility of conducting UK student led experiments in space via a UK company, thereby providing proof in principle that schools can commercially fly experiments within the UK/ESA.

\section{ACKNOWLEDGMENT}

This work was supported by the UK Space Agency and the Science and Technology Facilities Council [grant numbers ST/S006338/1 and ST/R005737]; and the Biotechnology and Biological Sciences Research Council [grant number BB/N015894/1].

\section{REFERENCES}

[1] NCESSE. Student spaceflight experiments program. (2019) Available at: http://ssep.ncesse.org/about-ssep/. 
[2] Osborn MJ. A Strategy for research in space biology and medicine in the new century. in Committee on SpaceBiology and Medicine, Space Studies Board, Commision on Physical Sciences, Mathematics, and Applications (Washington, D.C.: National Research Council, National Academy Press).

[3] Kern VD, Bhattacharya S, Bowman RN, Donovan FM, Elland C, Fahlen TF et al. Life sciences flight hardware development for the International Space Station. Adv Sp Res (2001) 27:1023-1030. doi:10.1016/S02731177(01)00178-8.

[4] Brinckmann E. Centrifuges and their application for biological experiments in space. Microgravity Sci Technol (2012) 24:365-372. doi:10.1007/s12217-012-9300-2.

[5] Warren P, Golden A, Hanover J, Love D, Shephard F, Szewczyk NJ. Evaluation of the fluids mixing enclosure system for life science experiments during a commercial Caenorhabditis elegans spaceflight experiment. Adv Sp Res (2013) 51:2241-2250. doi:10.1016/J.ASR.2013.02.002.

[6] Vukich M, Donati A, Zolesi V. Kayser Italia hardware for radiation and microgravity experiments in space. Rend Lincei (2014) 25:7-11. doi:10.1007/s12210-013-0261-1.

[7] ESA. Fly your thesis! / Education / ESA. 2019 Available at: https://www.esa.int/Education/Fly_Your_Thesis [Accessed July 23, 2019].

[8] Higashibata A, Szewczyk N, Conley C, Imamizo-Sato M, Higashitani A, Ishioka N. Decreased expression of myogenic transcription factors and myosin heavy chains in Caenorhabditis elegans muscles developed during spaceflight. J Exp Biol (2006) 209:3209-18. Available at: https://www.ncbi.nlm.nih.gov/pubmed/16888068.

[9] Szewczyk NJ, Tillman J, Conley CA, Granger L, Segalat L, Higashitani A et al. Description of International Caenorhabditis elegans experiment first flight (ICE-FIRST). Adv Sp Res (2008) 42:1072-1079. doi:10.1016/j.asr.2008.03.017.

[10] Gaffney CJ, Pollard A, Deane C, Cooke M, Balsamo M, Hewitt J et al. Worms in Space for Outreach on Earth: Space Life Science activities for the classroom. Gravitational Sp Res (2018) 6:2, 74-82. 